\title{
Constructing a Global and Integral Model of Business Management Using a CBR system
}

\author{
Emilio Corchado ${ }^{1}$, Juan M. Corchado ${ }^{2}$, Lourdes Sáiz ${ }^{1}$ and Ana Lara ${ }^{1}$ \\ ${ }^{1}$ Department of Civil Engineering, University of Burgos, Spain. \\ ${ }^{2}$ Departamento de Informática y Automática, University of Salamanca, Spain. \\ escorchardo@ubu.es, corchado@usal.es, lsaiz@ubu.es, amlara@ubu.es,
}

\begin{abstract}
Knowledge has become the most strategic resource in the new business environment. A case-based reasoning system, which incorporates a novel clustering and retrieval method, has been developed for identifying critical situations in business processes. The proposed method is based on a Cooperative Maximum Likelihood Hebbian Learning model, which can be used to categorize the necessities for the Acquisition, Transfer and Updating of Knowledge of the different departments of a firm. This technique is used as a tool to develop a part of a Global and Integral Model of business Management, which brings about a global improvement in the firm, adding value, flexibility and competitiveness. From this perspective, the model tries to generalise the hypothesis of organizational survival and competitiveness, so that the organisation that is able to identify, strengthen, and use key knowledge will reach a pole position.
\end{abstract}

Key words: Case-based Reasoning, Knowledge Management, hierarchical scheme and Cooperative Maximum Likelihood Hebbian Learning model.

\section{Introduction}

In this study, we centre our attention on the problem of knowledge management, from a pragmatic and managerial approach that contemplates, the possibility that knowledge can be classified and organised in order to achieve a better understanding. This issue is based, above all, on understanding the distinctions between transformations in forms of knowledge, starting from an inferior level (data and information) and advancing towards other higher levels, such as knowledge itself and its management, individual, and even organizational responsibilities.

This paper presents the results obtained with a case-based reasoning system (CBR) developed to identify critical situations that allow firms to take decisions about acquisition, transfer and updating processes in knowledge management. Case-based reasoning (CBR) systems have been successfully used in several domains such as diagnosis, monitoring, prediction, control and planning [9, 10, 11]. CBR systems require adequate retrieval and reuse mechanisms to provide successful results. Such 
mechanisms need to be consistent with the problem that has to be solved and with the data used to represent the problem domain. A CBR system is a methodology used to construct software tools to assist experts in the resolution of problems. The CBR system presented in this paper incorporates a Cooperative Maximum Likelihood Hebbian Learning model (CMLHL) that facilitates the data clustering and indexation and automates the retrieval and adaptation stages of the CBR system. This method is closely related to factor analysis (FA) and exploratory projection pursuit (EPP). It is a neural model based on the Negative Feedback artificial neural network, which has been extended by the combination of two different techniques. Initially by the selection of a proper cost function from a family of them, to identify the right distribution related to the data problem. This method is called Maximum-Likelihood Hebbian learning (MLHL) [3]. Then, lateral connections derived from the Rectified Gaussian Distribution [7] are added to the MLHL architecture [3]. These enforce a greater sparsity in the weight vectors.

This paper reviews the concept of CBR system and outlines the CMLHL model used in its construction. The first prototype of the system has been tested on a multinational group, specialised in the design and production of components for the automotive industry. This initial system is presented and the results obtained are shown.

\section{Case-based Reasoning Systems}

Case-based reasoning is used to solve problems by adapting solutions that were used to solve similar previous problems [11]. A case is normally composed of a number of attributes that represents a problem and of a solution for that problem. The operation of a CBR system involves the adaptation of old solutions to match new experiences, using past cases to explain new situations, using previous experience to formulate new solutions, or reasoning from precedents to interpret a similar situation.

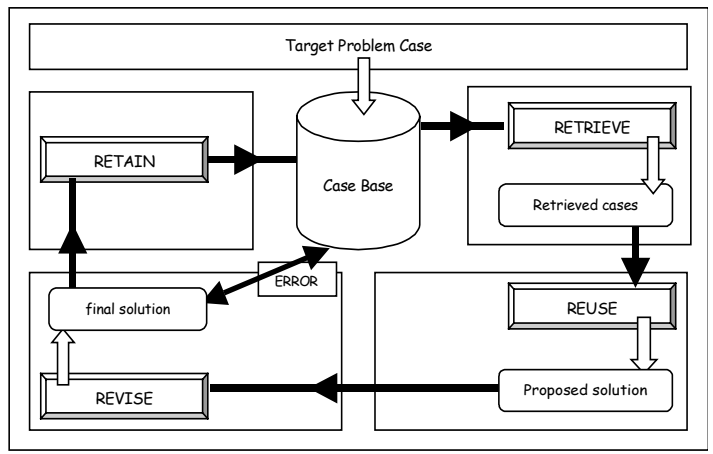

Fig. 1. CBR system reasoning cycle.

Fig 1. This figure shows the reasoning cycle of a typical CBR system that includes four steps that are cyclically carried out in a sequenced way: retrieve, reuse, revise, and retain [11]. During the retrieval phase, those cases that are most similar to the problem case are recovered from the case-base. 
The recovered cases are adapted to generate a possible solution during the reuse stage. The solution is then reviewed and, if appropriate, a new case is created and stored during the retention stage, within the memory. CBR systems update their casebases and consequently evolve with their environment. Although the CBR systems are tools to assist the decision taken process and they have not been developed to be autonomous, some of their reasoning stages may be automated $[8,9,10]$.

The CBR system developed in the framework of this experiment incorporates a CMLHL model that clusters the cases, facilitates the case indexation and automates the retrieval and adaptation stages. Now the CMLHL model is presented and then the proposed CBR system is outlined and evaluated.

\section{The Cooperative Artificial Neural Architecture.}

We use the standard Maximum-Likelihood Network [1, 3, 5] but now with a lateral connection (which acts after the feed forward but before the feedback) derived from the Rectified Gaussian Distribution [2, 6, 7] and using the cooperative distribution. Thus we have:

Feedforward:

$$
y_{i}=\sum_{j=1}^{\mathbf{N}} W_{i j} x_{j}, \forall i
$$

Lateral Activation Passing:

$$
y_{i}(t+1)=\left[y_{i}(t)+\tau(b-A y)\right]^{+}
$$

Feedback:

$$
e_{j}=x_{j}-\sum_{i=1}^{M} W_{i j} y_{i},
$$

Weight change:

$$
\Delta W_{i j}=\eta \cdot y_{i} \cdot \operatorname{sign}\left(e_{j}\right)\left|e_{j}\right|^{p-1}
$$

Where:

the parameter $\tau$ represents the strength of the lateral connections.

The cooperative distribution in the case of $\mathrm{N}$ variables is defined by:

$$
\begin{gathered}
A_{i j}=\delta_{i j}+\frac{1}{N}-\frac{4}{N} \cos \left(\frac{2 \pi}{N}(i-j)\right) \quad \text { and } \\
\mathrm{b}_{\mathrm{i}}=1
\end{gathered}
$$


where $\delta_{i j}$ is the Kronecker delta and $i$ and $j$ represent the identifiers of output neuron.

\section{Case study}

The developed system has been tested in a multinational group, leader in the design and production of a great variety of components for the automotive industry. The justification of this choice lies in the fact that the characteristics of its management represent a favourable environment and opportune moment for the introduction of Knowledge Management. There is an undergoing organizational change and the firm faces great growth and expansion, which requires a rapid adaptation to the demands of the sector, with greater resources, imminent transfers and accurate forecasting of knowledge, together with the immediate demand to capitalise on them, to share and to use them within the firm.

The design of the preliminary theoretical model of Knowledge Management shown if Fig.2 is based on three components: the Organisation -Strategy and People-, Processes -Acquisition, Transfer and Updating of Knowledge- and Technology Technological Aids-, from which the propositions of the model are defined.

The population sample used came to 277 registries (individuals) that correspond with the "necessities of knowledge" showed by the head of eleven departments of the company studied. This knowledge gathers different stages (knowledge levels) that depict the current situation of each department for the tasks or activities assigned to each department to be successfully accomplished. Also, it has been possible to obtain valuable data on the degree of importance for the company of the gathered knowledge. This way, it is possible to identify the lack of the knowledge that it is necessary to perform the activity, so as to make the right decision on its acquisition in terms of how it is acquired, or what is the cost or time needed. In the same way, it is possible to specify the knowledge possessed which is not comprehensively employed, either because the person does not use it in its entirely or because it has additional value and potential use, for other departments. Furthermore, it is possible to include the analysis corresponding to the necessary evolution of the present knowledge to detect new knowledge, to eliminate the obsolete one and to validate new necessities, among others. 


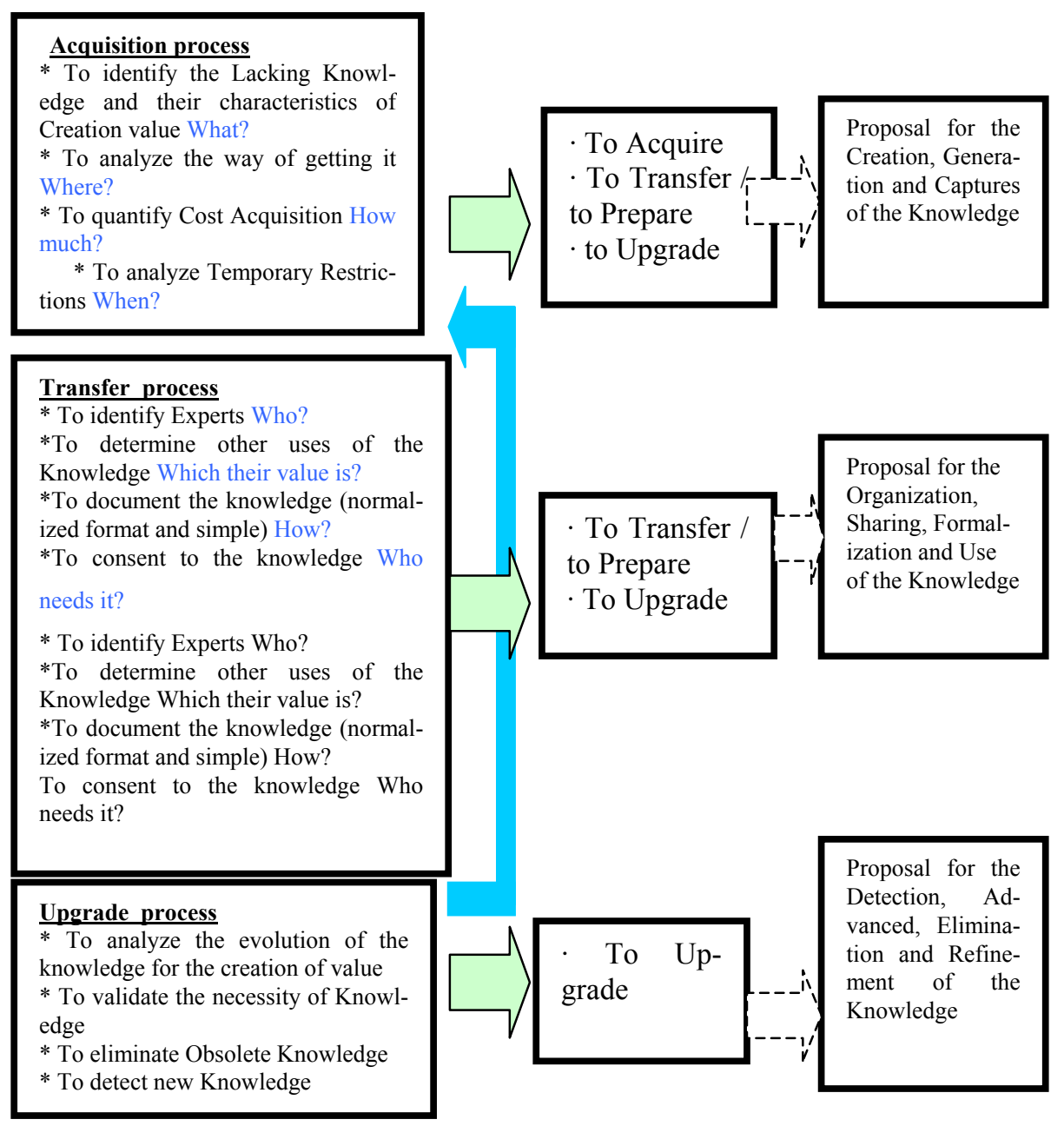

Fig. 2. Fig. 2 shows the theoretical model of Knowledge Management.

Then in this particular problem, the cases are composed of several attributes, representing the state of the enterprise, and the solution is an attribute that represents the situation of the firm and the degree of information required to improve its management process. The data was register by observations, questionnaires and personal interviews to the employees of the studied company. Figure 3 shows the results obtained in this study. The case-base stores 277 cases representing previous states of the firm together with their associated risk levels. Cases are clustered and indexed using the CMLHL model, as can be seen in Figure 3.a. The retrieval and 
the CMLHL model, as can be seen in Figure 3.a. The retrieval and reuse stage are carried out applying equations 1 to 4 . As a result of the application of these equations to a given problem case (a new situation of the firm), we obtain information about its risk level, which may be one of the stages presented in Figure 3.b. Figure 3.b is obtained by an analysis of the results obtained and shown in Fig. 3a. To see the relation between Fig3.a and Fig3.b, we have kept the same nomenclature. The CBR system is comparing a new situation with previously evaluated ones and letting us know at which group the present situation belongs. The revision is carries out manually in this first prototype and ones a new case is evaluated, it is incorporated to the case-base of the CBR system during the retain stage (learning step).

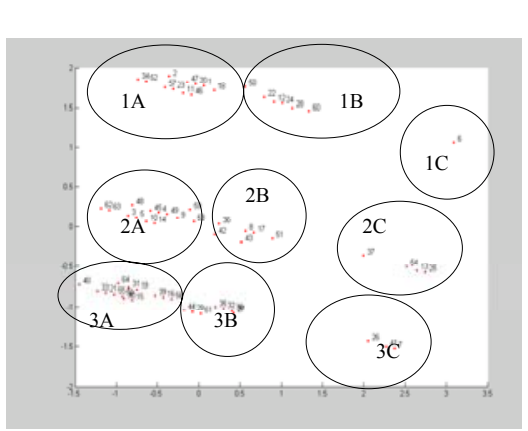

Figure 3.a: CMLHL on the real data.

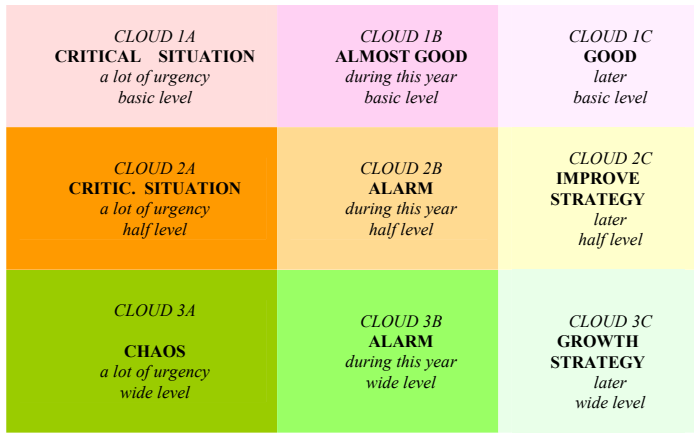

Figure 3.b: Results Representation.

Fig. 3. Fig.3.a shows the result of CMLHL clustering and indexing process on the case-base. The projection identifies separated clusters (clouds), each of then has been labeled. We have identified mainly 9 clusters or clouds. Fig. 3 b is a graphical representation of Fig.3a.

\section{Preliminary results}

The system presented above is an assistant tool. It has helped us to identify, in an automated way, the risk states at which a firm may be and to understand more about this business management process. In terms of firm type, the points of cloud $1 \mathrm{C}$ are related to a GOOD SITUATION. The firm is in this place because the level of knowledge required is low and therefore the acquisition of knowledge is not a priority. Also the fact that only one point (point 6 in Fig. 3a.) appears underlines the fact that the company only has to acquire knowledge in one specific area.

In a contrasting case, in the area occupied by the clouds labelled as $3 \mathrm{~A}$, there is a lot of urgency to acquire knowledge at a wide level. This area is called "CHAOS". In a similar way, in the area occupied by clouds $1 \mathrm{~A}$ and $2 \mathrm{~A}$ there is a need to acquire knowledge urgently at a half or basic level. It could be that in these cases there is a holding of knowledge that can put the company in a CRITICAL SITUATION, since it may depend on the concession of new projects, the incorporation of new clients and all those parameters that somehow help to generate activity within in the firm.

The area occupied by the points of the cloud $2 \mathrm{C}$ outlines the possibility to acquire knowledge at a later stage but in one period but at a half level. This could mean an 
IMPROVE STRATEGY in the firm, where it needs to improve in what it already possesses. However, cloud $3 \mathrm{C}$ represents the situation that the firm has to acquire the knowledge later but at a wide level. This means that the company should think about the idea of enlarging and growing, both in terms of new processes, and new products. This is: GROWTH STRATEGY. The points corresponding to area 1B are related to an ALMOST GOOD area, because the knowledge is needed urgently at a basic level. Cloud $2 \mathrm{~B}$ and $3 \mathrm{~B}$ identifies an ALARM area, because there is not urgency and the level needed is half.

The initial results show that the presented system is a reliable tool to identify critical situations that allow firms to take decisions about acquisition, transfer and updating processes about knowledge management. Other methods, such as Self Organizing Maps have been applied and have provided less accurate results, from the point of view of the firm management experts. CMLHL provides more sparse projections than the others methods $[6,12]$ and captures some type of global ordering in the data set. A second prototype of this system is under construction. It will be integrated with in the distributed management system of the enterprise and it will deal with more information about the firm.

\section{Acknowledgements}

This work has been supported by the Spanish Council for Science and Technology (MCYT) project TIC2003-07369-C02-02.

\section{References}

1. E. Corchado and C. Fyfe, Orientation Selection Using Maximum Likelihood Hebbian Learning, International Journal of Knowledge-Based Intelligent Engineering Systems Volume 7 Number 2, ISSN: 1327-2314. Brighton, United Kingdom. April (2003).

2. E. Corchado, Y. Han, C. Fyfe. Structuring global responses of local filters using lateral connections. J. Exp. Theor. Artif. Intell. 15(4): 473-487 (2003)

3. E. Corchado, D. MacDonald, and C. Fyfe, Maximum and Minimum Likelihood Hebbian Learning for Exploratory Projection Pursuit, Data mining and Knowledge Discovery, Kluwer Academic Publishing, (In press)

4. J. Friedman and J. Tukey. A Projection Pursuit Algorithm for Exploratory Data Analysis. IEEE Transaction on Computers, (23): 881-890. (1974).

5. C. Fyfe, and E. Corchado, Maximum Likelihood Hebbian Rules. European Symposium on Artificial Neural Networks. (2002)

6. E. Corchado, C. Fyfe: Connectionist Techniques for the Identification and Suppression of Interfering Underlying Factors. International Journal of Pattern Recognition and Artificial Intelligence. Vol. 17, No 8 (2003) 1447-1466.

7. H.S. Seung, N.D. Socci, and D. Lee, The Rectified Gaussian Distribution, Advances in Neural Information Processing Systems, 10. 350 (1998).

8. J. M. Corchado and J. Aiken (2002) Hybrid Artificial Intelligence Methods in Oceanographic Forecasting Models. IEEE SMC Transactions Part C. Vol. 32, No.4. pp. 307-313.

9. J. M. Corchado and B.Lees (2001) A hybrid case-based model for forecasting. Applied Artificial Intelligence: An International Journal Vol 15, no. 2, pp 105-127.

10. S. K.Pal, T. S. Dillon and D. S. Yeung (2000) Soft Computing in Case-based Reasoning. (eds.). Springer Verlag, London, U.K. 
11. I. Watson and F. Marir (1994) Case-Based Reasoning: A Review. Cambridge University Press, 1994. The knowledge Engineering Review. Vol. 9. №3.

12. T. Peña, L. Joyanes, M. A Manzanedo, L. Sáiz, Knowledge Management and measuring intellectual capital in business organisations, Soft Computing (SOCO'2001). UK 